\title{
The Myth of Reference Varieties in English Pronunciation across the Subcontinent, Egypt and Kingdom of Saudi Arabia
}

\author{
Muhammad Khan ${ }^{1}$ \\ ${ }^{1}$ Jazan University, Jazan, Kingdom of Saudi Arabia \\ Correspondence: Muhammad Khan, Jazan University, Jazan, Kingdom of Saudi Arabia. E-mail: \\ dr.muhammadkhan786@gmail.com
}

Received: March 28, 2015 Accepted: April 21, 2015 Online Published: May 30, 2015

doi:10.5539/ijel.v5n3p19 URL: http://dx.doi.org/10.5539/ijel.v5n3p19

\begin{abstract}
The present study aims at exploring the differences in pronunciation more or less prevailing in the Indian Subcontinent and Arab world with a special focus on Pakistan, India, Bangladesh, Saudi Arabia, and Egypt. The study identifies the most common factors that affect English pronunciation in general: (i) some phonemic differences that exist in L1 and English as a target language, (ii) Improper teaching and learning of English pronunciation. When non-native speakers of English exchange their ideas among themselves, their comprehension is to the maximum. But their pronunciation seems problematic in case the speaker or interlocutor is a native speaker. A test for the nationals of the lands included in the study was developed and administered to identify and specify the exact area(s) of pronunciation difficulties either consonants or vowels at segmental level of phonology. The analysis and conclusion of the test fully proved that English pronunciation is deeply influenced by the sound system of indigenous languages. As a matter of fact, English pronunciation of some non-native speakers, through their best possible efforts, may be closer to native speakers but not exactly like that of natives. The fact is that native and non-native differences in English pronunciation are unquestionable. Moreover, non-natives living in different areas (sometimes of the same community) also differ more or less in their pronunciation.
\end{abstract}

Keywords: reference varieties, English phonemic differences, segmental phonology, native and non-native, sound system, first language, second language, accent, variation

\section{Introduction}

Phonology both at segmental and supra-segmental levels is a complex phenomenon especially for non-native speakers of English which invites our attention to address the issue at all levels, particularly, in the context when a speaker and a listener experience intelligibility problems. Production and perception of sounds are the most important aspects towards a successful communication. A never ending research on English(es) of the world and its variety is going on but the findings are always divergent. A crystal clear fact is that the environment of education is the most vital factor towards the standard of non-native English and especially accent and pronunciation. But this does not solve all the problems related to non-native English accent. A plethora of research in the area of intelligibility at international level exists but all this could not put forward any solution to the problem of unintelligible varieties of English pronunciation. The researchers who oppose non-native English teachers and appreciate only native English teachers worldwide are actually ignoring the ground realities and no doubt their intentions are just sweeping statements. In fact the provision of native English teachers all over the world is not only difficult but impossible. The questions arise: (i) how many countries in the world are there which may financially afford native English teachers?; (ii) how many native English teachers are available to meet the world demand?; (iii) who will guarantee a single standard of English throughout the world?

Another important aspect of the problem is that even in America and UK, the variety of English accent and pronunciation is ever growing regionally and from one social class to the other. So the communication and comprehension within native English speaking world is not problem-free. The differences between native and non-native English speakers are obvious and natural that cannot be avoided. This does not mean that non-native English speakers' pronunciation should always go unchecked and diverged. The novel idea is that there cannot be a single standard of the world English(es) and its/their pronunciation. Again the question arises: whose English and which English should be determined as a 'standard'? The problems of intelligibility multiply when 
inevitable varieties of English across the globe are taken into account. If both 'purists' and 'moderates' ideas are accepted in their own right then native and non-native speakers and teachers should be given due consideration to promote a balanced approach instead of exaggeration in one or the other way. Here the important point is that variation in any language is a natural phenomenon which cannot be checked through man-made boundaries and limitations.

Another interesting trend is observed that a non-native English speaker tries his best to ignore his local accent and master native-like accent and the motive behind this effort may be employment, higher education, social status, so on and so forth. But even then he may not speak exactly like natives. The fact is that no speaker of the outer circle speaks RP and that even if he did, the speaker would definitely lack manners distinct of a native speaker of English language (Kachru, 1986, pp. 31-44). Obviously realizing the status of native accent in English speaking world, maximum students at college and university levels in the Subcontinent and Arab world prefer to use native varieties at the expense of their local varieties. However, the general atmosphere in the non-native milieu of that of acceptance of the local varieties and those who appreciate native-like pronunciation, are regarded as the exception rather than the rule. In a nutshell the problem of unintelligibility may be minimized if non-native English teachers and learners worldwide put in practice an approach of convergence from the very beginning. But this needs highly qualified and well trained English teachers at all levels.

\subsection{Conceptual Framework}

Generally British English and American English are regarded two main native varieties and these two can be the model for other varieties but this directly undermines and negates non-native speakers' cultural and sociolinguistic realities. The proponents of the Romantic Model (Prator, 1968, pp. 458-75; Quirk, 1990, pp. 5-15) are of the view that English as an international language should enjoy monolithic status. But on the other hand (Kachru, 1996, p. 241-250) emphasizes that the global spread of English language needs to be seen with multi-identities and in its regional and social context. The non-native varieties make English language more colourful and prove identity of various communities. The present situation demands reasonable tolerance on the part of the purists.

Moreover, Kachru is apt in saying that English as an international language should not be seen as the sole property of native English speakers. In the present era the number of non-native English speakers all over the world exceeds the number of native speakers. Kachru's idea has also been supported by (Pakir, 2010, p.109). She highlighted two distinctive factors of the global spread of English: firstly, "we have to place the English language in its regional and social context" and secondly "we have to accept the inevitable pluri-centricity of English instead of due-centricity viz the British and the American centres of linguistic hegemony". It means that the researchers who always favour only native speakers and teachers need to see the overall situation through the glasses of reality in its true perspective.

Moreover, Bamgbose (1998, pp. 3-14), commented that Quirk and Kachru were engaged in"a battle royal on Standard English as a norm for the English using world". Quirk favoured a global standard which he thought would guarantee international intelligibility whereas Kachru appreciated the legitimacy and equality of Englishes in different circles. Simo Bobda (2000, pp. 249-265) reported how in South Africa, the increasing divergence of South African Black English from the native English norms had taken place. Unfortunately despite the consensus by a majority of scholars that it is unrealistic to impose native norms in non-native settings, some conservatives still have the idea that British and American English should be used in non-native environment. Quirk, (1990, pp. 3-10) raises concerns about native and non-native variation: (i) this variation in pronunciation will wet up barriers to communication and (ii) "we won't be able to breakdown these barriers to careers and social mobility". On the other hand Stefan, (2008, pp. 9-29) thinks English the barrier and mobility as a prejudice against regional varieties. Kachru, (1992, pp. 4-12) as quoted by Pakir, (2010, pp. 109) reiterates that in fact the concept of intelligibility is a two sided issue where the native and non-native speaker were seen as equal partners in the communication process.

In addition to this, different studies on pedagogical problems exist: "The teacher's dilemma" (Platt et al., 1984), "World Englishes: to teach or not to teach" (Brown, 1977, pp. 179-190), "Torn between the norms" (Bamgbose, 1998, pp. 27-35), and so on. The researchers that oppose non-native teachers, give the argument that if native models and teachers are used in the non-native environment, this will add to the degree of intelligibility worldwide. On the other hand the presence of a non-native teacher will lead to the dissemination of local features and the dream of mutual intelligibility across the globe will be hindered. But such views have been rejected by some scholars advancing the argument that the use of native model in a non-native setting will definitely ignore the sociolinguistic and cultural realities of the non-native English learner. Moreover, Prator, (1968, pp. 458-75) 
and Chevillet, (1993, pp. 29-37); Christoperson, (1992, pp. 15-19); House, (1999, pp. 73-89) and Jenkins, (2000, pp. 39-47) are of the view that non-native speakers are not qualified to teach English language. The debates on "Non-native teachers teaching pronunciation" and "Native English teachers teaching pronunciation" are going side by side but all in vain. Simo Bobda, (1995, pp. 247-269) advances the idea that there are economic benefits in maintaining native model of English as the sole norm. It is Britain and America who can enjoy selling their English teachers and material unchallenged. In addition to this he quotes Sir Richard Francis, Director General of the British Council who comments that "Britain's real black gold is not the north sea oil but the English language". Similarly, the Director of the International House in London writes "once we used to send gunboats and diplomats abroad; now we are sending English teachers".

Now the real motive behind this issue is crystal clear and the point to be noted is that crucial to the problems faced by non-native speakers of English are the natives' attitude towards these new English varieties. Mc Arthur, (1992, pp. 12-18); Peter, (2003, pp. 43-46); Widdowson, (1997, pp. 133-141); Kershaw, (1996, pp. 7-11); Parrot, (1998, pp. 20-23) and Gnutzmann, (2000, pp. 355-361), are of the view that actually language is closely bound up with certain cultural and social identities and attitudes and certainly both native and non-native English speakers encounter varying attitudes towards varieties of a language. Realizing this fact, natives' idea of linguistic hegemony can be changed into positive attitude towards non-native varieties particularly in pronunciation and accent in general. The idea of variation in accent can further be strongly underpinned by the unquestionable reality of the Qur'anic accent where all tribes were unable to recite the Holy Qur'an in a single accent so in the beginning, recitation of the Word of Allah was allowed in seven accents. This regional variation in a language is some sort of natural phenomenon and beyond human control. Whatever the language is and whatever learner's commitment is, he cannot be exactly a native speaker, however, he can be 'closer to' or 'like' a native speaker. Again it is worth noting that pronunciation and general accent is not the preserve of only non-native varieties of English language. Being a natural linguistic phenomenon, even in the native environment like Britain and the USA, there are noticeable regional and social accents that often cause intelligibility problems which further goes down to 'idiolect' (for details see Wells, (1985, pp. 54-68); Crystal, (1999, pp. 10-20); Trask, (2001, pp. 35-73), Algeo, (1991, p. 254) and Brown, (1968, pp. 179-90. Roach, (2009, p. 163), comments on the issue go like, "It is all too easy to talk about such things as 'Scottish English', 'American English', and so on, and to ignore the variety that inevitably exists within any large community of speakers. Each individual's speech is different from any others ... the idea of a standard pronunciation is a convenient fiction, not a scientific fact. In a word both native and non-native English speakers need to compromise with maximum convergence and minimum divergence.

\subsection{Objectives}

Objectives of the study are to:

(i) determine how native and non-native English speaker differ in pronunciation,

(ii) identify the causes of difference in non-native English pronunciation,

(iii) clarify whether the slight variations in pronunciation result in communication problems,

(iv) Suggest ways for English teachers and the learners how to minimize pronunciation differences for better communication and listening comprehension.

\section{Research Methodology and Data Collection}

Research tools play a vital role in research. Therefore, a test was developed for the selected non-native English speakers to determine their differences in pronunciation of some selected English words in isolation (see appendix A). The examinees include non-native English speakers from: (a) Bangladesh, (b) Egypt, (c) India, (d) Pakistan and (e) Saudi Arabia. For a representative sample 50 speakers each were given the test. Firstly, except a few, mostly the pronunciation test was administered on line through recording. The participants were asked to articulate 55 words in a natural way as they generally pronounce them and no careful pronunciation. The test was completed in four sittings on different dates because of time constraint and ultimately completing the target of 220 words in total. Their pronunciations were recorded on a computer with a well-adjusted sensitive microphone. The data collected through this research tool, was analyzed with utmost care with encouraging results.

\subsection{Date Analysis}

The data collected through English pronunciation test was analyzed meticulously, ensuring validity and reliability of the study: 
The English pronunciation test administered to the target population from Bangladesh (BDE), Egypt (EE), India (IE), Pakistan (PE) and Saudi Arabia (SAE) was analyzed and its result displayed in two different tables such as: table-1 (Consonants) and table-2 (vowels).

Table 1. (Consonants): Bangladesh English (BDE), Egypt English (EE), India English (IE), Pakistan English (PE), Saudi Arabia English (SAE) and Natives English (NE). For natives pronunciation 'Oxford Advance Learner Dictionary' has been used.

\begin{tabular}{|c|c|c|c|c|c|c|}
\hline Word & $\mathrm{BDE}$ & $\mathrm{EE}$ & $\mathrm{IE}$ & $\mathrm{PE}$ & SAE & $\mathrm{NE}$ \\
\hline abashed & $\mathrm{t}$ & $t$ & d & d & $t$ & $\mathrm{t}$ \\
\hline abrasion & z & $d \overline{d z}$ & $z$ & 3 & $d \bar{d}$ & 3 \\
\hline$p$ anel & $\mathrm{p}$ & $b$ & $p$ & $p$ & $\mathrm{~b}$ & $p$ \\
\hline handle & d & d & $d$ & $d$ & d & $d$ \\
\hline judaism & z & $\int$ & $\widehat{d z}$ & $\widehat{d z}$ & s & $\widehat{d z}$ \\
\hline asthma & $\int$ & s s & z & s & s $\int$ & $s / z$ \\
\hline author & d & s & $\theta$ & $\theta$ & s & $\theta$ \\
\hline autumn & $\mathrm{mn}$ & $\mathrm{mIn}$ & $\mathrm{mn}$ & $\mathrm{mn}$ & min & $\mathrm{m}$ \\
\hline awful & ph & $f$ & $f / p$ & $f$ & $f$ & $f$ \\
\hline bargain & g & k & g & g & k & g \\
\hline barrage & z & $\int$ & $\widehat{d_{3} / z / \int}$ & $\sqrt[d 3]{3}$ & $\int$ & 3 \\
\hline bomb & $\mathrm{mb}$ & mib & $\mathrm{mb}$ & $\mathrm{mb}$ & mib & $\mathrm{m}$ \\
\hline calcium & s & $\int$ & $\int$ & $\int$ & $\int$ & s \\
\hline charisma & $\widehat{d z}$ & z & $\overline{d_{3} / z}$ & $z$ & z & z \\
\hline chew & s & s & ts & $\widehat{\mathrm{t} J}$ & s & tf \\
\hline chocolate & $\mathrm{s}$ & s & ts & $\widehat{\mathrm{t} J}$ & $\int$ & ts \\
\hline $\operatorname{close}(\mathrm{V})$ & $\widehat{d z}$ & z & $\widehat{d_{3} / z}$ & $z$ & z & z \\
\hline clothes & z/ ठ & z & z/ ð & ð & z & ð \\
\hline clumsy & $\int$ & s & $\widehat{d z} / z$ & s & s & z \\
\hline colonel & kəlunəl & kəlunəl & kəlunəl & kəlunəl & kəlunəl & k3:nl \\
\hline consumption & $\int$ & $\int$ & $\int / \mathrm{s}$ & z & $\int$ & s \\
\hline consume & $\widehat{d z}$ & $\int$ & $\overline{d_{3} / z}$ & z & $\int$ & s \\
\hline coup & $\mathrm{f}$ & b & $p / f$ & $p$ & $\mathrm{~b}$ & $\mathrm{P}$ (silent) \\
\hline crew & kəru: & krru: & kəru: & kəru: & kIru: & Kru: \\
\hline cricket & kIr & $\mathrm{kIr}$ & kIr & kIr & kIr & krI \\
\hline enough & $\mathrm{p} / \mathrm{ph}$ & $f$ & $p / f$ & $f$ & $f$ & $f$ \\
\hline faithful & $\operatorname{thh} / \mathrm{z}$ & z & $\delta / z$ & ð & z & $\theta$ \\
\hline fashionable & $s / \int$ & $\int$ & $s / \int$ & $\int$ & $\int$ & $\int$ \\
\hline festival & bh & $f$ & v & v & $f$ & v \\
\hline garage & $\mathrm{z}$ & $\int$ & $d_{3} / z$ & $d \overline{d z}$ & $\int$ & 3 \\
\hline genre & $\mathrm{z}$ & s & $\widehat{d z} / z / \int$ & $\widehat{d z}$ & s & 3 \\
\hline giant & z & $d \overline{d z}$ & $d_{3}$ & $\widehat{d z}$ & $d \bar{d}$ & $\widehat{d z}$ \\
\hline gentleman & $\mathrm{z}$ & $\widehat{d z}$ & $\overline{d z} / z$ & $\widehat{d z}$ & $\widehat{d z}$ & $\widehat{d z}$ \\
\hline heir & $\mathrm{h}$ & $\mathrm{h}$ & $\mathrm{h}$ & $\mathrm{h}$ & $\mathrm{h}$ & e \\
\hline lease & $\int$ & $\int$ & $z$ & $z$ & $\int$ & s \\
\hline novelty & $\mathrm{b}$ & $f$ & v & v & $f$ & v \\
\hline pizza & $\overline{d z}$ & z & $\widehat{d} d_{3} / z$ & z & z & tsa \\
\hline
\end{tabular}




\begin{tabular}{|c|c|c|c|c|c|c|}
\hline reason & $\widehat{d z} / z$ & z & $\widehat{d z} / z$ & z & z & z \\
\hline sadism & $\mathrm{t}$ & $d$ & $d$ & $d$ & $d$ & $d$ \\
\hline session & $\int$ & $\int$ & $\int / \mathrm{s}$ & $\mathrm{s}$ & $\int$ & $\mathrm{s}$ \\
\hline tangible & $\mathrm{z}$ & $\widehat{d z}$ & $\widehat{d z} / z$ & $\widehat{d z}$ & $\widehat{d z}$ & $\widetilde{d} 3$ \\
\hline theft & $\mathrm{t}$ & $\mathrm{s}$ & $t / s / \theta$ & $\theta$ & $\mathrm{s}$ & $\theta$ \\
\hline through & $t^{h}$ & $\mathrm{~s}$ & $\theta / \mathrm{s}$ & $\theta$ & $\mathrm{s}$ & $\theta$ \\
\hline vision & $\mathrm{z}$ & $\int$ & $\int / \mathrm{z} / 3$ & 3 & $\int$ & 3 \\
\hline wear & bhh & $f$ & w & w & $f$ & w \\
\hline provoke & bhh & b & $p$ & $p$ & $b$ & $\mathrm{p}$ \\
\hline neglect & $\mathrm{z} / \mathrm{g}$ & $\mathrm{g} / \mathrm{k}$ & g & g & k & g \\
\hline
\end{tabular}

\section{Description table 1:}

Table 1 shows the differences in consonant sounds in English pronunciation of the selected words in the target population. The table contains words in the first column with differences in their pronunciation in columns 2-7 in Bangladeshi English (BDE), Egyptian English (EE), Indian English (IE), Pakistani English (PE), Saudi Arabian English (SAE) and Natives English (NE). For different consonants sounds in pronunciation, different words have been included that definitely added to the reliability and validity of the study. Moreover, the required consonants in the words have been italicized. Then instead of complete pronunciation of the words only the differences in the italicized consonants sounds specifically in pronunciation of the selected population have been given for the ease of the reader.

\section{Explanation table 1:}

Well-documented research evidence in the area of differences in pronunciation from community to community and even within community is a hard job. However, some examples of the variation in pronunciation of certain English words in Bangladesh, Egypt, India, Pakistan, Saudi Arabia and Native speakers have been determined for a general impressionistic evaluation. The most common consonants sound differences in English pronunciation of the target population, as shown in the table, giving only one word (for each variation) as an example with reason of variation, are as follows:

(a) Natives and Bangladeshis pronunciation differences
Word
Difference
(a) abrasion
$/ 3 / \rightarrow / z /$
$\underline{\text { Reason }}$
(b) giant
$/ \mathrm{dz} / \rightarrow / \mathrm{z} /$
(c) Consumption
$/ \mathrm{s}, \mathrm{z} / \mathrm{s} / \mathrm{\int} /$
(d) theft
$/ \theta / \rightarrow / \mathrm{d}^{\mathrm{h}} / \mathrm{t}^{\mathrm{h}} /$
(e) enough
$/ \mathrm{f} / \rightarrow / \mathrm{p}^{\mathrm{h}} /$
(f) chew
tf $\rightarrow / \mathrm{s} /$
(g) reason
$\mid z, s / \rightarrow / \widetilde{d z} /$
L1 sound interference
(h) clothes
$/$ / $\rightarrow / z /$
(i) provoke
$/ \mathrm{p} / \rightarrow / \mathrm{b}^{\mathrm{h}} /$
L1 sound interference
(j) crew
$/ \mathrm{kr} / \rightarrow$ /kər/
Lack of teaching-learning of English pronunciation
(k) cricket
$/ \mathrm{krI} / \mathrm{h} / \mathrm{kr} /$
Local sound differences
(l) fashion
$/ \mathrm{S} / \rightarrow \mathrm{s} /$
Local sound differences
(m) very
$/ \mathrm{v} / \rightarrow / \mathrm{b}^{\mathrm{h}} /$
L1 sound interference
(n) heir
/e/ $\rightarrow / \mathrm{h} /$
L1 sound interference
(o) sadism
$/ \mathrm{d} / \rightarrow / \mathrm{t} /$
L1 sound interference
Local sound differences
Consonants declusterization
(p) wear
$/ \mathrm{v} / \rightarrow / \mathrm{b}^{\mathrm{h}} /$
Consonants declusterization
(q) neglect
$/ \mathrm{g} / \rightarrow / \mathrm{z} /$
L1 sound interference
Local sound differences
Spelling pronunciation
L1 sound interference
Local sound differences
L1 sound interference 
(b) Natives and Egyptian/Saudi Arabian pronunciation differences

Word

(a) abashed Difference

(b) abrasion

$/ \mathrm{t} / \rightarrow / \mathrm{t} / \mathrm{emphatic}$

(c) panel

$/ 3 / \rightarrow / \mathrm{d} 3 / \mathrm{J} /$

(d) handle

$/ \mathrm{p} / \rightarrow / \mathrm{b} /$

(e) Judaism

$/ d / \rightarrow / d /$ emphatic

(f) clumsy

$/ \mathrm{d} z / \rightarrow / \mathrm{s} /$

(g) author

$\mid \mathrm{z} / \rightarrow / \mathrm{s} /$

(h) autumn

$/ \theta / \rightarrow / \mathrm{s} / \mathrm{z} /$

(i) bargain

$/ \mathrm{mn} / \rightarrow$ /min/

(j) calcium

$/ \mathrm{g} / \rightarrow / \mathrm{k} / \mathrm{g} /$

(k) chew

$/ \mathrm{s} / \rightarrow / \mathrm{S} /$

(l) clothes

/t $/ \rightarrow / \mathrm{d} /$

(m) crew

$/ \mathrm{d} / \rightarrow / \mathrm{z} /$

(n) cricket

$/ \mathrm{kr} / \rightarrow$ kər/

(o) wear

(p) heir

$/ \mathrm{krI} / \mathrm{h} / \mathrm{krr} /$

$/ \mathrm{v} / \rightarrow / \mathrm{f} /$

/e/ $\rightarrow$ /h/

(c) Natives and Indian pronunciation differences

Word

(a) abashed

Difference

(b) abrasion

$/ \mathrm{t} / \rightarrow / \mathrm{d} /$

(c) consume

$/ 3 / \rightarrow / z / \int / \widehat{d} 3 /$

(d) bomb

$/ \mathrm{s} / \rightarrow / \mathrm{d} z / \mathrm{z} /$

(e) awful

$/ \mathrm{m} / \rightarrow / \mathrm{mb} /$

(f) close

$/ \mathrm{f} / \rightarrow / \mathrm{p} /$

(g) clothes

$/ \mathrm{z} / \mathrm{d} / \mathrm{d} /$

(h) coup

$/$ / $\rightarrow$ /z/

(i) crew

('p' silent) $\rightarrow / p / f /$

(j) cricket

/kr/ $\rightarrow$ /kər/

(k) theft

$/ \mathrm{krI} / \mathrm{h} / \mathrm{krr} /$

(l) fashion

$/ \theta / \rightarrow / \mathrm{s} /$ ð/z/t/

(m) heir

$/ \mathrm{s} / \mathrm{s} /$

/e/ $\rightarrow / \mathrm{h} /$
Reason

No ' $t$ ' sound in Arabic

L1 sound interference

No ' $p$ ' sound in Arabic

No 'd' sound in Arabic

L1 sound interference

L1 sound interference

No such sound in Arabic

Consonant declusterization

No ' $g$ ' sound in Arabic

L1 sound interference

No / $\mathrm{t}$ / sound in Arabic

L1 sound interference

Consonant declusterization

Consonant declusterization

L1 sound interference

Spelling pronunciation

$\underline{\text { Reason }}$

Lack of teaching-learning of English pronunciation

L1 sound influence

L1 sound influence

Spelling pronunciation

L1 sound interference

L1 sound influence

L1 sound influence

Spelling pronunciation

Consonant declusterization

Consonant declusterization

L1 pronunciation effect

L1 sound interference

Spelling pronunciation 
(d) Natives and Pakistani pronunciation differences

$\begin{array}{lll}\text { Word } & \text { Difference } & \underline{\text { Reason }} \\ \text { (a) abashed } & / \mathrm{t} / \rightarrow / \mathrm{d} / & \text { Lack of teaching-learning of English Pronunciation } \\ \text { (b) autumn } & / \mathrm{m} / \rightarrow / \mathrm{mn} / & \text { Spelling pronunciation } \\ \text { (c) barrage } & / \mathrm{z} / \rightarrow / \mathrm{d} / / & \text { Lack of teaching-learning of English pronunciation } \\ \text { (d) calcium } & / \mathrm{s} / \rightarrow / \mathrm{J} / & \text { Lack of teaching-learning of English pronunciation } \\ \text { (e) asthma } & / \mathrm{z} / \rightarrow / \mathrm{s} / & \text { Lack of teaching-learning of English pronunciation } \\ \text { (f) consume } & / \mathrm{s} / \rightarrow / \mathrm{z} / & \text { Lack of teaching-learning of English pronunciation } \\ \text { (g) crew } & / \mathrm{kr} / \rightarrow / \mathrm{k} r / & \text { Lack of teaching-learning of English pronunciation } \\ \text { (h) cricket } & / \mathrm{krI} / \rightarrow / \mathrm{krr} / & \text { Consonant declusterization } \\ \text { (i) through } & / \theta / \rightarrow / \mathrm{d} / & \text { L1 pronunciation influence } \\ \text { (j) heir } & / \mathrm{e} / \rightarrow / \mathrm{h} / & \text { Spelling pronunciation }\end{array}$

Note. The symbol $(\rightarrow)$ denotes 'changes into'.

Table 2. (Vowels): Bangladesh English (BDE), Egypt English (EE), India English (IE), Pakistan English (PE), Saudi Arabia English (SAE) and Natives English (NE). For natives pronunciation 'Oxford Advance Learner Dictionary' has been used.

\begin{tabular}{|c|c|c|c|c|c|c|}
\hline ablution & $\int \partial n$ & Sən & $\int ə n$ & Sən & Sən & jn \\
\hline accurate & e & I & eI & eI & I & a \\
\hline abnormal & mal & məl & məl & mal & mal & $\mathrm{ml}$ \\
\hline abrasion & zan & dzən & zən & zən & dzən & $3 n$ \\
\hline accountable & abəl & Ibəl & erbəl & eibəl & Ibəl & əbl \\
\hline accuracy & $\mathrm{rafI}$ & rISI & reisi & reisi & rISI & rəsi \\
\hline accumulate & ka-mv & ka-mv & ka-mv & ka-mv & ka-mv & kju:-mju: \\
\hline April & $\mathrm{e}$ & I & $\mathrm{e}$ & $\mathrm{e}$ & I & eI \\
\hline ardent & $\mathrm{e}$ & I & $\mathrm{e}$ & $\mathrm{e}$ & I & $\mathrm{dnt}$ \\
\hline $\arg u m e n t$ & U-I & $U-I$ & $U-I$ & $U-I$ & $U-I$ & ju: - ə \\
\hline articulate & eI & I & eI & eI & I & ə \\
\hline artless & $\mathrm{e}$ & I & $\mathrm{e}$ & $\mathrm{e}$ & I & I \\
\hline author & a: & a: & a: & a: & a: & ग: \\
\hline awful & fol & fol & fol & fol & fol & $\mathrm{fl}$ \\
\hline bargain & e & I & ег & ег & I & I \\
\hline basically & kəlı & kəlı & kəlı & kəlı & kəli & klI \\
\hline bestow & bis & bis & bes & bes & bis & bis \\
\hline bomb & $\mathrm{ba}$ & bv & $b_{\wedge}$ & $b_{\wedge}$ & bv & bo \\
\hline breath(N) & $\mathrm{rI}$ & rI & ri: & ri: & rI & re \\
\hline calamity & I & I & I & I & I & ə \\
\hline cautious & IV & I & Іә & Іә & I & ə \\
\hline charisma & a & $æ$ & $æ$ & $æ$ & $æ$ & ə \\
\hline colonel & nal & nəl & nəl & nəl & nəl & $\mathrm{nl}$ \\
\hline comedy & $a--e$ & U-I & U--e & U--e & U-I & D--ə \\
\hline crescent & sent & sint & sent & sent & sint & snt \\
\hline crew & kəru: & kIru: & kəru: & kəru: & kIru: & kru: \\
\hline
\end{tabular}




\begin{tabular}{|c|c|c|c|c|c|c|}
\hline cricket & IkIrI & kIrI & kIrI & kIrI & kIrI & krI \\
\hline curtain & $a-ə$ & $\Lambda-\mathrm{I}$ & $\Lambda-\partial$ & $\Lambda-\partial$ & $\Lambda-\mathrm{I}$ & 3:-tn \\
\hline devil & e-ə & $I-\partial$ & e-ə & e-ə & I-ə & $e-v l$ \\
\hline endless & e-e & $I-I$ & e-e & e-e & I-I & e-I \\
\hline faithf $u l$ & eI-v & $æ-\mho$ & eI-v & eI- - & $æ-\mho$ & eI-fl \\
\hline festival & e-I-ə & I-I-ə & e-I-ə & e-I-ə & I-I-ə & $e-\partial-v l$ \\
\hline judaism & $\mathrm{u}:-\mathrm{a}-\mathrm{I}$ & $\mathrm{u}:-\mathrm{a}-\mathrm{I}$ & $\mathrm{u}:-\mathrm{a}$ - I & $\mathrm{u}:-\mathrm{a}-\mathrm{I}$ & $\mathrm{u}:-\mathrm{a}-\mathrm{I}$ & u: - eI-I \\
\hline many & $æ$ & $æ$ & $æ$ & $æ$ & æ & e \\
\hline mountain & av-e & $U-I$ & av-e & av-e & $U-I$ & av-I \\
\hline neglect & $e-e$ & $I-I$ & $e-e$ & $e-e$ & I-I & I-e \\
\hline novelty & əठ-I & $U-\mathrm{I}$ & әひ-e & әЈ-e & $U-I$ & $\mathrm{D}-\mathrm{vl}$ \\
\hline old & əठ & $v$ & $\partial U$ & $\partial U$ & $v$ & əઇ \\
\hline panel & e- ə & I-ə & æ-ə & æ-ə & I-ə & $æ-\mathrm{nl}$ \\
\hline pair & еə & Іә & еіә & еіә & Іə & еə \\
\hline provoke & $a-v$ & U-U & əઇ-əひ & əひ-əひ & U-U & Ә-əひ \\
\hline reason & ә & ə & ә & ә & ә & $\mathrm{zn}$ \\
\hline said & $æ$ & $æ$ & $æ$ & $æ$ & $æ$ & e \\
\hline saturate & æ-u:-eI & æ-U-I & æ-ひ-eI & æ-ひ-eI & æ-U-I & æ-ə-eI \\
\hline selection & I-e-U & I-I-ə & e-e-ə & e-e-ə & I-I-ə & I-e-fn \\
\hline series & i: & I & i: & i: & I & Іә \\
\hline session & $æ$ & I & eI & eI & I & e \\
\hline shovel & $a$ & $v$ & a: & $\mathrm{a}:$ & U & $\wedge$ \\
\hline sociology & $\mho-\mho$ & U-ə & əU-əU & ӘU-əઇ & U-ə & əひ-ə \\
\hline solace & əひ-eI & U-I & əU-eI & əU-eI & $U-\mathrm{I}$ & $D-I$ \\
\hline tangible & e-I-ə & $\mathrm{I}-\mathrm{I}-\partial$ & e-I-ə & e-I-ə & I-I-ə & æ-ə- \\
\hline territory & e-I-ə & I-I-I & e-I-ə & e-I-ə & I-I-I & e-ə- \\
\hline vigilant & e & I & $\mathrm{e}$ & $\mathrm{e}$ & I & ә \\
\hline vocal & әU & $v$ & əઇ & əઇ & $v$ & $\partial \mho$ \\
\hline wear & I & I & I & I & I & еə \\
\hline widow & $\partial \mho$ & v & əठ & $\partial U$ & v & $\partial \mho$ \\
\hline xerox & $I-U$ & $\mathrm{I}-U$ & i:-əઇ & i:-əひ & $I-U$ & Iə-D \\
\hline zenith & I & I & I & I & I & $\mathrm{e}$ \\
\hline
\end{tabular}

\section{Description table 2:}

Table-2 reflects the differences in vowels sounds in English pronunciation of the selected words in the target population. The selected words have been displayed in the first column of the table with differences in their pronunciation in columns 2-7 in Bangladeshi English (BDE), Egyptian English (EE), Indian English (IE), Pakistani English (PE), Saudi Arabian English (SAE) and Natives English (NE). For different vowel sounds in pronunciation, different words have been included that definitely added to the reliability and validity of the study. Moreover, the required vowels in the words have been italicized. Then instead of complete pronunciation of the words only the differences in the italicized vowels sounds specifically in pronunciation of the selected population, have been given for easy and quick comprehension.

\section{Explanation table 2:}

Research on the differences in English pronunciation particularly vowel sounds from community to community and even within community throughout the world is the most demanding one. However, some examples of the 
variation in pronunciation of certain English words in Bangladesh, Egypt, India, Pakistan, Saudi Arabia and Native speakers have been determined for a partial depiction of the problem. The most common vowel sound differences in English pronunciation of the target population, as displayed in the table, giving only one word (for each variation) as an example with reason of variation, are as follows:

(a) Natives and Bangladeshis pronunciation differences
Word
Difference
Reason
(a)ablution
$\int \mathrm{n} \rightarrow \int \partial \mathrm{n}$
L1 interference
(b)accurate
rət $\rightarrow$ rAt
Local language
(c) accountable
əbl $\rightarrow$ Ibəl
Local accent
(d)curtain
k3:tn $\rightarrow$ katən
Local accent
(e)accumulate
$\mathrm{kju}: \rightarrow \mathrm{ka}$
L1 interference
(f) $A$ pril
eI $\rightarrow$ e
Lacks pronunciation teaching
(g)ardent
dnt $\rightarrow$ dent
Lacks pronunciation teaching
(h)argument
$\partial \rightarrow \mathrm{I}$
(i) articulate
$\partial \rightarrow$ eI
Lacks pronunciation teaching
(j) artless
$\mathrm{I} \rightarrow \mathrm{e}$
Local accent
(k)author
ว: $\rightarrow$ a:
Local accent
(l) awful
$\mathrm{fl} \rightarrow \mathrm{fol}$
Lacks pronunciation teaching
(m) basically
klı $\rightarrow$ kəli:
L1 and Local accent
(n) Bomb
$\mathrm{bp} \rightarrow \mathrm{ba}$
L1 and local accent
(o) Breath
re $\rightarrow$ ri:
Lacks pronunciation teaching
(p) Cautious
$\partial \rightarrow$ IU
Lacks pronunciation teaching
(q) Comedy
$\mathrm{p} \rightarrow \mathrm{a}:$
(r) Crescent
snt $\rightarrow$ sent
(s) Many
$\mathrm{e} \rightarrow æ$
L1 and local accent
(t) Solace
$\mathrm{D} \rightarrow$ əU
Lacks pronunciation teaching
(u) Reason
zn $\rightarrow$ zən
L1 and local accent
(v) Series
гә $\rightarrow$ i:
Local accent
(w) Shovel
$\wedge \rightarrow$ a:
Lacks pronunciation teaching
L1 and local accent
Lacks pronunciation teaching
L1 and local accent

(b) Natives and Egyptian/Saudi Arabian pronunciation differences

Word

(a) Ablution

(b) Accurate

(c) Many

(d) Abrasion

(e) Accumulate

(f) April

(g) Ardent

(h) Argument

(i) $\boldsymbol{A u}$ thor

(j) Awful

(k)Basically

(1) Comedy
Difference

$\int \mathrm{n} \rightarrow \int ə \mathrm{n}$

ə $\rightarrow$ I

e $\rightarrow$ I/æ

3n $\rightarrow$ 3ən

kju: $\rightarrow$ ka

eI $\rightarrow$ I

dnt $\rightarrow$ dint

jv: $\rightarrow$ v

ग: $\rightarrow$ a:

$\mathrm{fl} \rightarrow \mathrm{fol}$

kli $\rightarrow$ kəli

$\mathrm{D} \rightarrow \mathrm{v}$
Reason

L1 interference

L1 accent

Lacks pronunciation teaching

L1 sound system

L1 pronunciation

L1 sound system

L1 influence

Lack pronunciation teaching

Lacks pronunciation teaching

L1 influence

L1 influence

L1 sound system 

(m) Crew
kru: $\rightarrow$ kIru:
Consonat-declusterization
(n) Curtain
3: $\rightarrow \wedge$
(o) Judaism
eI $\rightarrow$ I/a
Lack pronunciation teaching
(p) Sociology
əठ $\rightarrow$ U
L1 sound system
(q) Tangible
$æ \rightarrow$ I
L1 sound system
(r) Said
$\mathrm{e} \rightarrow æ$
L1 sound system
(s) series
Iə $\rightarrow \mathrm{i}$ :
Lacks pronunciation teaching
(t) Shovel
$\wedge \rightarrow$ v
L1 pronunciation
(u) Wear
eə $\rightarrow$ I
Lacks pronunciation teaching
L1 sounds/accent

(c) Natives and Indian/Pakistan pronunciation differences
$\underline{\text { Word }}$
Difference
Reason
(a) Ablution
$\int \mathrm{n} \rightarrow \int ə \mathrm{n}$
L1 sound system
(b) Accurate
$\partial \rightarrow$ eI
(c) Accumulate
kju: $\rightarrow$ ka
Lacks pronunciation teaching
(d) April
eI $\rightarrow$ e
Lacks pronunciation teaching
(e) Ardent
dnt $\rightarrow$ dent
Lacks pronunciation teaching
(f) Argument
jv: $\rightarrow \mathrm{u}:$
(g) Artless
$\mathrm{I} \rightarrow \mathrm{e}$
(h) Author
ग: $\rightarrow$ a:
L1 sound system
(i) Awf $\boldsymbol{u l}$
$\mathrm{fl} \rightarrow \mathrm{fol}$
Lacks pronunciation teaching
Lacks pronunciation teaching
(j) Basically
kli $\rightarrow$ kəli
L1 sound system
(k) Provoke
$\partial \rightarrow \partial v$
L1 accent
(l) Solace
$\mathrm{D} \rightarrow \partial U$
L1 accent
(m) Session
e $\rightarrow$ eI
(n) Vigilant
$\partial \rightarrow \mathrm{e}$
L1 accent
(o) Crescent
snt $\rightarrow$ sent
L1 sound system
(p) $\mathrm{Crew}$
kru: $\rightarrow$ kəriv
Lacks pronunciation teaching
(q) Curtain
3: $\rightarrow \wedge$
(r) Judaism
eI $\rightarrow$ ə
Local accent
Local accent
(s) Many
$\mathrm{e} \rightarrow æ$
(t) Pair
еә $\rightarrow$ егә
Spelling pronunciation
(u) Series
гә $\rightarrow$ i:
Lacks pronunciation teaching
Lacks pronunciation teaching
Lacks pronunciation teaching
Lacks pronunciation teaching
(v) Shovel
$\wedge \rightarrow$ a:
Local accent
(w) Tangible
$æ \rightarrow \mathrm{e}$
Lacks pronunciation teaching
(x) Wear
еə $\rightarrow$ Іә
Lacks pronunciation teaching
Local accent

\section{Discussion and Conclusion}

In the context of the Sub-continent that includes Pakistan, India and Bangladesh, Egypt and Saudi Arabia, the differences in English accent are crystal clear. These variations due to one or the other reason are natural and inevitable. It is easy to talk about the reference varieties (standard varieties) in English pronunciation but aspiring for the monolithic approach throughout the world seems a dream. The variations in English pronunciation going on in the countries ibid have been concluded as follows:

(a) Bangladesh: In Bangladesh local language varieties deeply affect English pronunciation. Some of the reasons of mispronunciation include:

(i) Bangali English learners are generally used to spelling pronunciation. 
(ii) English sounds are not focused and emphasized.

(iii) They don't differentiate long and short vowels.

(iv) Bangla language has almost twenty plosives as compared to only six plosives of English which definitely interfere.

(v) The pronunciation of Bangali English speakers are entirely influenced by their mother tongue and local variety.

(vi) Another reason of differences is that English fricatives - f, $\theta, \partial, v, z, 3$, do not exist in Bangla inventory of phonemes.

(vii) Alveolar /t/ and /d/ are sometimes retroflexed by the Bangali speakers from the South region.

(b) India and Pakistan: Indian and Pakistani English speakers have more or less similar English pronunciation except some specific states, like Kerila, Utter Pradesh and Behar. The most distinctive differences in English pronunciation are:

(i) The consonants ' $\mathrm{v}$ ' and ' $\mathrm{w}$ ' in the words like 'very' and 'work' are pronounced similarly. Whereas in articulation ' $\mathrm{w}$ ' is bilabial approximant and ' $\mathrm{v}$ ' is labiodental fricative.

(ii) The alveolar/t/ and /d/ are often heard with drum-like heavy sound.

(iii) In some states of India like Behar, the /J/ sound is often heard with lisp /s/, e.g. the word 'sheikh' is pronounced as /seikh/.

(iv) There is no clear distinction between the vowel sounds $/ \mathrm{D} /$ and $/ \mathrm{J} /$. Both sound like $/ \mathrm{a} /$.

(v) Particularly in India 'retroflex' sounds are common in Telugu, Kannada, Tamil and Malayalam.

(vi) The word like 'class', 'staff' and 'last' are usually pronounced with a back /a/ as in Southern British dialects but unlike Northern British.

(vii) The voiceless 'plosives' /p/, /t/ and / k/ are not aspirated by Pakistani and Indian English speakers.

(viii) In native Indian languages except Tamil, the distinction between aspirated and un-aspirated plosives is phonemic.

(ix) In some of the Indian languages, there are two entirely distinctive sets of coronal plosives; one is 'dental' and the other is 'retroflex'. Moreover, to the Indian ears, the English alveolar plosives sound more retroflex than dental.

(x) In some parts of Utter Pradesh and Behar, the consonant /s/ preceding alveolar/t/ allophonically changes to $/ \mathrm{J} /$. For example, the word 'stop' is pronounced as $/ \mathrm{fta}: \mathrm{p} /$.

(xi) The $/ 3 /$ sound changes into /z/ or /dz/ by many Indian speakers and in Sound India this sounds like / $/$ /

(xii) Sanskrit and Marathhi have /s/ and /// sounds but Bangla, Udiya and Hindi do not.

(xiii) Moreover, Indian and Pakistani English speakers are more or less rhotic.

(xiv) English alveolar /t/ and /d/ are often retroflex / $/$ / and $/ \mathrm{d} /$, especially in South of India. But Hindi does not have true 'retroflex plosives' whereas Tamil speakers sound retroflexive.

(xv) Indian and Pakistani English speakers usually change syllabic $/ 1 /, / \mathrm{m} /$ and $/ \mathrm{n} /$ into [əl], [əm] and [ən].

(xvi) Pakistani and Indian English speakers often use light/clear/1/ like Irish English.

(xvii) Spelling pronunciation is also common in Pakistan and India.

(c) Egypt and Saudi Arabia: Except a few colloquial variations in English pronunciation, Egyptians and Saudi Arabians English speakers have similar accent. Some of the salient differences in Egyptian and Saudi English speakers include:

(i) The sound $/ z /$ which is reduction of $/ \bar{d} z /$ sound of Egyptianized loan words, tend to merge with [ $\left.\int\right]$; for example, the word 'garage' is mostly pronounced as /gara:// even by educated speakers.

(ii) The sound $/ \theta /$ and $/ ð /$ are always approximated to sibilants $[\mathrm{s}]$ and $[\mathrm{z}]$. Traditionally the interdental consonants correspond to the Egyptian Arabic alveolar-consonants [ t ] and [ d ].

(iii) Egyptians and Saudis use extra vowel sound in consonant clusters. For example, the words - 'split', 'throw' and 'lengths are pronounced as /spilitt/, /IOrəo/ and /leyðIs/ respectively. 
(iv) Some speakers focus on voicing and devoicing of some consonants, e.g. voiced [ $\mathrm{t} \rightarrow \mathrm{d}],[\mathrm{s} \rightarrow \mathrm{z}],[\mathrm{k} \rightarrow$ $\mathrm{g}],\left[\mathrm{3} \rightarrow \int\right]$ and $[\mathrm{v} \rightarrow \mathrm{f}]$.

(v) Egyptian English speakers also have problems with the vowels like [ I, , e, ə, 3, æ, ^ and ว ].

(vi) There are some problematic consonants for Egyptian and Arab speakers For example, [ $p \rightarrow b$ ], [ $3 \rightarrow \int$ ], $[\mathrm{r} \rightarrow \mathrm{r}+\mathrm{r}][\theta \rightarrow \mathrm{s}]$ and $\left[\mathrm{t} \rightarrow \int\right]$.

(vii) Moreover, [ p ], [ b ], [ d ] and [g ] sounds don't exist in Arabic inventory of phonemes. For example, the words - 'panda', 'police', and 'very', are pronounced as /ba:nda/, /bəoli:s/ and /feri/ respectively.

(viii) The words like 'pit', 'pet', 'put', 'pot', and 'pat', etc are difficult to pronounce with marginal distinction by Arab speakers. The words that are differentiated by the vowel sounds are a bit problematic for them.

\section{Suggestions}

In the light of the discussion and conclusion of the study it is quite clear that dreaming reference variety (standard pronunciation) of English across the globe needs revisiting the undeniable fact of naturally inevitable variations in English pronunciation. Particularly the native speakers of English language (which is already a major lingua franca in the world of today would) have to decide and compromise as to what is possible and impossible to do. Both native and non-native speakers of English should practise what is possible and at the same time compromise to the area of predictable and unavoidable difficulties. These are the problems of English pronunciation which are impossible to overcome for the Second Language Learners throughout the world even if they try their best. However, maximum efforts in teaching-learning of English pronunciation should be extended especially focusing upon:

(a) Teaching-learning of English pronunciation needs to be given priority at early stage because the interference of $\mathrm{L} 1$ becomes to its maximum at some later stages.

(b) Second language learners' English pronunciation may not be exactly like native speakers but they should try their best to be as closer to the natives as possible.

(c) The interference/influence of local languages of non-natives is not so easy to eradicate but it can be minimized through teaching-learning of English pronunciation and then practicing with a sense of self-improvement.

(d) For better listening comprehension, regular listening to native speakers will definitely prove beneficial and productive for Second Language Learners.

(e) The alphabet/sounds which do not exist in L1 of the learners of English as L2 need efforts to practice certain sound of the target language.

(f) First of all the most specific areas of difficulties in teaching-learning of English pronunciation should be identified and then focused constantly for effective teaching-learning of the pronunciation.

\section{References}

Algeo, J. (1991). The Origins and Development of English Language. UK: Oxford University Press.

Bamgbose, A. (1998). Torn Between the Norms! Innovations: World Englishes. Oxford and USA: Blackwell Publishers.

Baumgardner, R. J. (1996). South Asian English: Structure, Use and Users. USA: University of Illinois Press.

Bobda, A. S. (2000). Comparing some phonological features across African accents of English. English Studies. Berlin: Peter Lang.

Brown, K. (1977). Listening to Spoken English. London: Longman.

Chevillet, F. (1993). English or Englishes? English Today, 9(4). Cambridge: Cambridge University Press.

Dollinger, S. (2008). New Dialect Formation in Canada. USA: John Benjamins Pub. Co. http://dx.doi.org/10.1075/slcs.97

Gnutzmann, C. (2000). Lingua Franca. In Bryam, M. (Ed.), The Routledge Encyclopedia of Language Teaching and Learning. London: Routledge

House, J. (1999). Misunderstanding in Intercultural Communication: Interactions in English as a Lingua Franca and the Myth of mutual intelligibility. In Gnutzmann, C. (Ed.), Teaching and Learning English as a Global Language. Tübingen: Stauffenburg Pub.

Jenkins, J. (2000). The Phonology of English as an International Language. Oxford: Oxford University Press. 
Kachru, B. B. (1986). The Alchemy of English: The Spread, Functions and Models of Non-Native Englishes. Oxford: Pergamon Press.

Kachru, B. B. (1990). World Englishes and Applied Linguistics: World Englishes. Oxford: Pergamon. http://dx.doi.org/10.1075/z.lkul2.19kac

Kachru, B. B. (1996). The Paradigms of Marginality: World Englishes. Washington DC: Georgetown University Press.

Kershaw, G. (1996). The developing roles of native-speaker and non-native-speaker teachers. Modern English Teacher, 5(3).

McArthur, T. (1992). Models of English. English Today. Cambridge: Cambridge University Press.

Pakir, A. et al. (2010). Asian Englishes Today: English in Singapore Modernity and Management. Hong Kong: Hong Kong Uni. Press.

Peter, L., Wolf, H. G., \& Bobda, A. S. (2003). An account of distinctive phonetic and lexical features of Gambian English. English World Wide. Berlin-Frankfurt: Peter Lang.

Prator, C. (1968). British Heresy in TESL: Language Problems in Developing Nations. New York: John Wiley.

Quirk, R. (1990). Language Varieties and Standard Language: English Today. Delhi: Orient Paperbacks.

Roach, P. (2009). English Phonetics and Phonology: A Practical Course. UK: Cambridge Uni. Press.

Trask, R. L. (2001). Introducing Linguistics. UK: Totem Books pub.

Wells, J. C. (1985). English Accents in England. UK: Cambridge Uni. Press.

Widdowson, H. G. (1997). EIL, ESL, EFL: global issues and local interests. World Englishes, 16(1). http://dx.doi.org/10.1111/1467-971X.00054

\section{Appendix A}

\section{Pronunciation Test of Some Selected English Words in Isolation}

\begin{tabular}{|c|c|c|c|c|c|c|}
\hline Word & Bangladesh & Egypt & India & Pakistan & Saudi Arabia & Natives \\
\hline abacus & abakas & abakes & abcekəs & abcekəs & aba:kes & cebəkəs \\
\hline abashed & abaft & abafit & abasəd & abæed & $a b æ \int t$ & abæ $\int t$ \\
\hline ablution & ablu:fən & ablufən & ablu:sən & ablu:fən & ablu:fən & əblu: $\int \mathrm{n}$ \\
\hline abnormal & abna:rməl & Ibnarməl & ebnarməl & ebnarməl & ^bnarməl & æbn॰:ml \\
\hline abrasion & ^brazən & Ibridżən & ebreiz/dzzn & ebreizən & Ibreidzəzon & obre $13 n$ \\
\hline abuse(V) & abu: $\widehat{d 3}$ & Ibju:z & әbju:dz & әbju:z & Ibju:z & əbju:z \\
\hline abuse(N) & abu: $\overline{d z}$ & Ibju:z & әbju:dz & әbju:z & Ibju:z & əbju:s \\
\hline abysmal & əbid̋zməl & əb I zməl & əbid̋zməl & ob I sməl & əb I zməl & əb I zməl \\
\hline academic & akatemık & Ikədımık & əkædəmık & əkædəmık & əkədImik & ækədem Ik \\
\hline academician & Ikatmifən & IkədimIfn & əkædmIfn & əkædmIJən & əkədimifən & əkædəm I $\int \mathrm{n}$ \\
\hline accessory & eksesuri & aksisri & esesuri & esesuri & aksisri & əksesər I \\
\hline accountable & Ikanteibəl & Ikuntibəl & Ikəunterbəl & Ikəunterbəl & Ikuntıbəl & əkauntəbl \\
\hline accumulate & akamu:Irt & Ikamu:Itt & Ikamu:leit & Ikamu:leit & Ikamu:Irt & əkju:mjole It \\
\hline accuracy & ekura:si: & IkUrisI & ækureisi: & ækuressi: & IkUrisI & ækjərəs I \\
\hline accurate & ekuret & Ikurit & ækureIt & ækureit & Ikurrt & ækjərət \\
\hline accursed & Ik3:st & Ik^rsid & Ik3:rsd & Ik3:rsd & Ik^rsid & ək3:sid \\
\hline apology & əfaluzai & Ibalvdु'I & Ipalu $\widehat{d}_{3} \mathrm{I}$ & Ipaludु' & Ibalu $\widehat{d}$ II & әppləđЗુ I \\
\hline appreciable & eprIfebəl & IbrifIbəl & eprIfreibəl & eprifreibəl & IbrIfIbəl & әpri: $\int ə \mathrm{bl}$ \\
\hline April & əbril & Ibril & æpril & æpril & Ibril & e I prol \\
\hline aptitude & epti:siod & Ibtrtu:t & æpti:tıud & æpti:tıod & Ibtrtu:t & æpt Itju:d \\
\hline
\end{tabular}




\begin{tabular}{|c|c|c|c|c|c|c|}
\hline ardent & a:rtent & ardinit & a:rdent & a:rdent & ardinit & $\mathrm{a}: \mathrm{dnt}$ \\
\hline argument & a:rgumint & arku:minit & a:rgu:mint & a:rgu:mint & arku:minit & a:gjomənt \\
\hline arm & a:rm & a:rm & a:rm & a:rm & a:rm & $\mathrm{a}: \mathrm{m}$ \\
\hline arrears & eiriərdz & IrIərz & eirıərdz & eirıərz & IrIərz & orioz \\
\hline articulate(A) & a:rtikjulet & a:rtıkulit & a:rtıku:leit & a:rtıku:leit & a:rtıkulit & a:tı kjolat \\
\hline articulate(V) & a:rttkjulet & a:rtıkulıt & a:rtıku:leit & a:rtrku:lert & a:rtıkulıt & a:trkjulert \\
\hline artless & a:rtles & a:rtlis & a:rtles & a:rtles & a:rtlis & a:tl is \\
\hline asked & $\mathrm{a}: \int \mathrm{kd}$ & a:skid & a:skd & a:skd & a:skid & a:skt \\
\hline assault & Ifaa:It & Isa:Ist & æsa:It & æsa:It & Isa:Ist & วsว:1t \\
\hline associate & efufi:ert & IsUfIeIt & æsufIeIt & æsひfIeIt & Isvfreit & $\partial \operatorname{səo} \int \mathrm{I} I \partial t$ \\
\hline asthma & aftəmə & IstImə & æsӨəmə & æsӨəmə & IstImə & æs/zmə \\
\hline asylum & æji:ləm & ISI/Am & esarləm & esailəm & ISIl/Am & əsa I ləm \\
\hline attitude & ati:tu:t & ItIt tjud & æti:tjud & æti:tjud & ItIt jud & æt I tju:d \\
\hline assist & $\partial \int \mathrm{I} \int \mathrm{t}$ & ISISIt & $\partial \int \mathrm{I} \int \mathrm{t}$ & əsist & ISISIt & əsISt \\
\hline audible & a:trbəl & a:dIbəl & a:dibəl & a:dibəl & a:dIbəl & ๑:dəbl \\
\hline author & a:dər & a:sər & a:Өər & a:Өər & a:sər & ว:Өə囚 \\
\hline automobile & a:tumubail & a:tumubil & a:tumubail & a:tumubail & a:tumubil & ə:təməbi:1 \\
\hline authority & əta:rəti: & әsa:rtI & əӨaa:rrti: & әӨaa:rrti: & әsa:rtI & 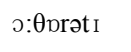 \\
\hline autumn & a:təmn & a:təmin & a:təmn & a:təmn & a:təmin & ৩:təm \\
\hline awful & əvful & əful & əvp/ful & əvful & əful & ॰:fl \\
\hline ball & ba:l & bol & ba:l & ba:l & bol & bo:1 \\
\hline balloon & balu:n & bilu:n & bælu:n & bælu:n & bilu:n & bəlu:n \\
\hline bamboo & bambu: & $b \wedge m b v$ & bəmbu: & bəmbu: & b^mbv & bæmbu: \\
\hline bargain & bargen & barkein & ba:rgein & ba:rgein & ba:rdzein & ba:g In \\
\hline barrage & bera:z & bira: $\int$ & bæra:dz/z & bæra:dz & bira: & bæ/əra:3 \\
\hline basically & besıkəli: & bIsIkəli: & beisıkəli & beisıkəli & bIsIkəli: & be Is Ikli \\
\hline basin & besin & bisin & beisin & beisin & bisin & be Isn \\
\hline bastion & bestion & bIstI-In & bestiən & bestiən & bIstI-In & bæst I ən \\
\hline bath(N) & ba:t & ba:s & ba: $\theta$ & ba: $\theta$ & be:s & ba:/æ $\theta$ \\
\hline bathe(V) & ba:t & bæz & ba: $\theta$ & ba: $\theta$ & bæz & bе Ið \\
\hline beautiful & brti:ful & bitIful & bivti:ful & bivti:ful & bitIful & bju:t Ifl \\
\hline beneath & bi:ni:t & bini:s & bi:ni: $\theta$ & bi:ni: $\theta$ & bini:s & b Ini: $\theta$ \\
\hline beneficent & binepisent & binIfISInt & binefi:sent & binefi:sent & binifisint & binefisnt \\
\hline beret & bIrrt & birrt & berit & berit & birrt & bere I \\
\hline bestow & bestu & bIstu & bestəu & bestəu & bIstu & bistav \\
\hline blessed(A) & bleft & bilisid & blesd & blesd & bilisid & blesid \\
\hline scissors & $\int \mathrm{I} \int \partial \widehat{d z}$ & sisərz & sisərz & sIsərz & sisərz & Si səZ \\
\hline blue-berry & blu:beri: & bilu:biri & blu:beri: & blu:beri: & bilu:biri & blu:bri \\
\hline bomb & ba:mb & bumb & $b \wedge m b$ & $b \wedge m b$ & bumb & bom \\
\hline bouquet & bukit & bukit & bu:keI & bu:ker & bukit & brke I \\
\hline breadth & bret & brits & bred $\theta$ & bred $\theta$ & brits & $\operatorname{bret} \theta$ \\
\hline breath $(\mathrm{N})$ & bri:t & birts & bri: $\theta$ & bri: $\theta$ & birrs & bre $\theta$ \\
\hline breathe $(\mathrm{V})$ & bri:t & bri:z & bri: $\theta$ & bri: $\theta$ & bri:z & bri:ð \\
\hline
\end{tabular}




\begin{tabular}{|c|c|c|c|c|c|c|}
\hline brook & bru:k & birok & bru:k & bru:k & biruk & brok \\
\hline burial & buriəl & buriəl & bərıəl & bərıəl & buriəl & beriəl \\
\hline cachet & kasit & $k \wedge \int \mathrm{I}$ & kæseI & kæJeI & $\mathrm{k} \wedge \int \mathrm{I}$ & $\mathrm{kæ} \int \mathrm{e}_{\mathrm{I}}$ \\
\hline calamity & kalamiti: & k^limItI & kəlæmıti: & kəlæmıti: & k^limItI & kəlæmət I \\
\hline calcium & kelfIəm & kIIJIəm & kelfIəm & kelfrəm & kIl|İm & kæls I əm \\
\hline camel & kæməl & k^məl & kæməl & kæməl & k^məl & $\mathrm{kæml}$ \\
\hline caption & kæffən & k^bJən & kæps/Jən & 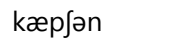 & k^bJən & $\mathrm{kæp} \int \mathrm{n}$ \\
\hline career & kærıər & k^rıər & kærıər & kærıər & k^rıər & kərIə(r) \\
\hline cassette & keisət & kIsIt & keIsIt & keIsit & kisit & kəset \\
\hline carnal & karnəl & ka:rnəl & ka:rnəl & ka:rnəl & ka:rnəl & ka:nl \\
\hline catharsis & katarfI & kIsa:rsis & kæӨa:rsız & kæӨa:rsız & kIsa:rsis & kəӨa:sis \\
\hline cautious & ka:frə] & ka:[I^s & ka:frəs & ka:fıəs & ka:[I^s & $\mathrm{k} \supset: \int ə s$ \\
\hline channel & sænəl & f^nəl & tfænəl & ţænəl & $\int \wedge n ə l$ & țænl \\
\hline charisma & kærıdzmə & k^rIzmə & kærıdzmə & kærızmə & k^rIzsmə & kərı zmə \\
\hline chasm & kædzəm & $\mathrm{k} \wedge \mathrm{ZIm}$ & kædzəm & kæzəm & $\mathrm{k} \wedge \mathrm{ZIm}$ & kæzəm \\
\hline chew & siu: & Ju: & $\widehat{\mathrm{t} f \mathrm{IU}}$ & tfIU & Ju: & ţu: \\
\hline chocolate & sa:kleit & fa:klit & tfa:kleit & tfa:kleit & fa:klit & $\mathfrak{t} \int \mathrm{pkl} l \partial t$ \\
\hline christian & krisiən & kIrt JIn & krist]Iən & krist]Iən & kIrIJIn & krist]ən \\
\hline climb & kəlaimb & IkIlaimb & kəlaimb & kəlaimb & IkIlaimb & kla Im \\
\hline close(adj) & kələudz & IkIləuz & kələud] & kələuz & IkIləuz & klaus \\
\hline $\operatorname{close}(\mathrm{V})$ & kələudz & IkIləひz & kələudz & kələひz & IkIləひz & kləoz \\
\hline $\operatorname{cloth}(\mathrm{N})$ & Ikla:t & kIlus & kəla: $\theta$ & kəla: $\theta$ & kIlus & $\mathrm{k} \ln \theta$ \\
\hline clothes(N) & Ikla:t & kIluzIz & kəla: $\theta$ & kəla: $\theta$ & kiluziz & kləuðz \\
\hline clothe(V) & Ikləud & kIləuz & kələひð & kələひð & kIləuz & kləuð \\
\hline clumsy & kəlımsi: & kIləmsI & kələmdżi: & kələmsi: & kIləmsI & $\mathrm{kl} \Lambda \mathrm{mzI}$ \\
\hline coast & kəひft & kust & kəust & kəust & kust & kəust \\
\hline coat & kəut & kvt & kəut & kəut & kvt & kəot \\
\hline cogent & kvdzent & kudzint & kəudzent & kəudzent & kudzint & kəuđЗ̋ənt \\
\hline colonel & kəlunəl & kəlunəl & kəlunəl & kəlunəl & kəlunəl & k3:nl \\
\hline Word & Bangladesh & Egypt & India & Pakistan & Saudi Arabia & Natives \\
\hline colony & ka:lu:nI & kaluni & ka:lu:ni: & ka:lu:ni: & kaluni & kplən I \\
\hline comedian & kumætiən & kumidən & kumediən & kumediən & kumidən & kəmi:d I ən \\
\hline comedy & kumeti: & kumidI & kumedi: & kumedi: & kUmIdI & kpməd I \\
\hline concise & k^nfaIdz & kunsaiz & kınsaIdz & kınsaiz & kunsaIz & kənsa Is \\
\hline conspiracy & kənfprIfI & k^nsbərisI & kənspireisI & kənspireisI & k^nsbərisI & kənsp I rəs I \\
\hline consumption & kəndzəmpfən & k^nzəmbfən & kənz^mp」ən & kənz^mpfən & kınzəmbJən & $\operatorname{kəns} \Lambda m p \int \mathrm{n}$ \\
\hline consume & kənḑzom & kınziUm & kənd̄jju:m & kənzju:m & kınziUm & kənsju:/u:m \\
\hline corruption & kur^ffən & kər^bJən & kurıpfən & kur^pJən & kər^bJən & $\operatorname{kər} \Lambda \mathrm{p} \int \mathrm{n}$ \\
\hline cough & $k \wedge p^{h}$ & $k \wedge f$ & $k \wedge f / p^{h}$ & $k \wedge f$ & $k \wedge f$ & $\mathrm{kD} / \mathrm{\rho}: \mathrm{f}$ \\
\hline coup & ku:f/p & kvb & ku:p & ku:p & kub & ku: \\
\hline couple & kəp/fəl & k^bəl & kәрәl & kәрәl & k^bəl & $\mathrm{k} \wedge \mathrm{pl}$ \\
\hline crescent & Ikresint & kIrIsint & kri:sent & kri:sent & kIrisint & kresnt \\
\hline crew & kəriu & kIru & kərju: & kərju: & kIro & kru: \\
\hline
\end{tabular}




\begin{tabular}{|c|c|c|c|c|c|c|}
\hline cricket & kIrkit & kIrIkIt & kIrkit & kIrkit & kIrIkIt & krikit \\
\hline criminal & kriminal & kIrıminəl & kriminal & kriminəl & kIrıminəl & kriminl \\
\hline crisis & kəraisidz & kIraisiz & kəraisiz & kəraisiz & kIraIsIz & krais is \\
\hline crises & kəraisidz & kIraisiz & kəraisiz & kəraisiz & kIraisiz & kra I si:z \\
\hline crystal & kərIstəl & kIrIstəl & kəristəl & kərIstəl & kIrIstəl & kristl \\
\hline curtain & kərtən & k^rtin & k3:rtən & k3:rtən & k^rtin & $\mathrm{k} 3: \mathrm{tn}$ \\
\hline correct & kurekt & kərIkt & kurekt & kurekt & kərikt & kərekt \\
\hline defiance & $\operatorname{dep}^{\text {h}}$ Ien $\int$ & drffins & defiens & defiens & drfrins & di fa I əns \\
\hline denouement & denumint & dinumint & dinumint & dinumint & dinumint & de Inu:møy \\
\hline exult & eksəlt & Iksılt & exs3:It & exs3:It & Iksslt & IgZnlt \\
\hline devil & dævəl & divNl & devəl & devəl & divnl & devl \\
\hline divorce & daibəur & darfurs & daIvəurs & darvəurs & darfurs & divo:s \\
\hline endless & endle & Indlis & endles & endles & Indlis & endlis \\
\hline eligible & ælıd్తżbəl & Ilıd̋̄Ibəl & elıḑ̄Ibəl & elıdð̄Ibəl & Ilıḑ3ıbəl & el I đदुəbl \\
\hline enough & enəp ${ }^{h}$ & In^f & enəp/f & enəf & InAf & In $\wedge f$ \\
\hline faithful & feitful & fidfol & fer$\theta f u l$ & ferӨful & fidfol & $\mathrm{fe}_{\mathrm{I}} \theta \mathrm{fl}$ \\
\hline fashionable & feifənibəl & fi Jənibəl & ferfənibəl & feifənibəl & fIJənibəl & $\mathrm{fæ} \int \mathrm{n} ə \mathrm{bl}$ \\
\hline festival & feftrvəl & fistivəl & festi:vəl & festi:vəl & fistivəl & festəvl \\
\hline finite & finit & finit & finit & finit & finit & fainait \\
\hline \multirow[t]{2}{*}{ garage } & geira:z & giraf & gæra:d3 & gira: $\overline{d 3}$ & giraf & gæ/əra:3/ \\
\hline & & & & & & gærıd3 \\
\hline garden & ga:rdən & dza:rdən & ga:rdən & ga:rdən & dza:rdən & ga:dn \\
\hline genre & za:nrI & $\int \wedge n r I$ & $\widehat{d} 3 /$ zanrr & 3a:nri & $\int \wedge n r I$ & 3a:nrə \\
\hline generic & zenrık & ginrik & z/dzanrık & dzanrtk & dzanrtk & d3̧ Iner $\mathrm{k} k$ \\
\hline giant & dziant & gi^nt & dżia;nt & dzia;nt & dziant & đद̧a Iənt \\
\hline gentleman & dzentəImæn & gintəlmin & zentəImæn & dzentəImæn & d3Intəlmin & dక̄entlmən \\
\hline Word & Bangladesh & Egypt & India & Pakistan & Saudi Arabia & Natives \\
\hline handle & hændəl & h^ndil & hændəl & hændəl & h^ndil & fændl \\
\hline heath & hi:t & hi:s & hi: $\theta$ & hi: $\theta$ & hi:s & hi: $\theta$ \\
\hline hebrew & hebriv & IbIru: & IbriU & hibriv & Ibiru: & hi:bru: \\
\hline heir & heiər & Iər & heiər & heiər & Iər & eə(r) \\
\hline judaism & dzu:təIdzəm & dzodiIzəm & dzu:dərzəm & dzu:dərzəm & dzudiızəm & dЗ̧u:de I I zm \\
\hline lease & li: $\int$ & li:s & li:z & li:z & li:s & li:s \\
\hline $\operatorname{limb}$ & IImb & IImb & $\operatorname{limb}$ & IImb & IImb & $\lim$ \\
\hline many & mæni & $\mathrm{m} \wedge \mathrm{nI}$ & mæni: & mæni: & m^nI & men I \\
\hline menace & mInæe & $\min \wedge s$ & mInæs & minæs & $\min \wedge s$ & menos \\
\hline mountain & məunten & muntin & məunten & məunten & muntin & maunt in \\
\hline mobile & mubail & mubail & mubail & mubail & mubail & məuba Il \\
\hline neglect & neglect & nIglikit & neglect & neglect & niḑ̧lıkıt & niglekt \\
\hline noise & na:Iz & na:Iz & na:Iz & na:Iz & na:Iz & nวIz \\
\hline nose & nəudz & nuz & nəudz & nəひz & nuz & nəoz \\
\hline novelty & nəひvelti: & nuvilti & nəชvelti: & nəuvelti: & nUVIltI & novlt I \\
\hline nude & nuvilti & nud & nju:d & nju:d & nud & nju:/u:d \\
\hline
\end{tabular}




\begin{tabular}{|c|c|c|c|c|c|c|}
\hline ocean & 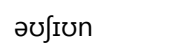 & 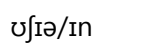 & əUs/JIən & 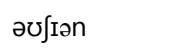 & Ufזə/In & $\partial 0 \int \mathrm{n}$ \\
\hline offensive & әupensib & vfinsif & əof/pensiv & əofensiv & vfinsif & ofensiv \\
\hline old & əolt & vlid & ovld & ovld & vlid & oold \\
\hline panel & pænəl & p^nəl & pænəl & pænəl & p^nəl & pænl \\
\hline provoke & provəəuk & provovk & prəuvəư & prəuvəuk & provok & prəvəəuk \\
\hline pigeon & pidżın & bIdzin & pIz/dżəən & pidżiən & bId̄3in & pid3̆ın \\
\hline pizza & pi:đzə & bi:zə & pi:z/dzəə & pi:zə & bi:zə & pi:tsə \\
\hline quarrel & kvərl & kvəril & kvərl & kvərl & kuərIl & kwprəl \\
\hline raisin & reIfin & rIsIn & reisin & reisin & risin & re izn \\
\hline ravine & $\operatorname{rab}^{h}$ In & rifin & reivin & reivin & rIfIn & rəvi:n \\
\hline refusal & riphju:zəl & rifjuzəl & rrfju:z/dzəol & rIfju:zəl & rrfjuzəl & rifju:zl \\
\hline reason & ri:ฮ̧dzən & rIZIn & ri:z/dżən & ri:zən & rizin & ri:zn \\
\hline receivable & rısi:brbəl & risivibəl & rIsi:verbəl & rIsi:verbəl & risivibəl & risi:vabl \\
\hline recompense & rikımpenf & rikəmbinis & rakəmpens & rakəmpens & rikəmbinis & rekəmpens \\
\hline resistance & ridżidztenf & riziztins & riziztens & rIziztens & riziztins & riziztəns \\
\hline responsible & respanfibəl & risbansibəl & respansibəl & respansibəl & risbansibəl & risponsəbl \\
\hline rival & rarbh & raifəl & raivəl & raivəl & raIfəl & ra Ivl \\
\hline riven & raibhən & rIfən & rIvən & rivən & rIfən & rivn \\
\hline ruin & roin & roin & roi:n & roi:n & roin & ru: In \\
\hline sacred & sækrrt & sıkrid & sækrıd & sækrıd & s^krid & seIkrid \\
\hline sadism & sædəIdz̋əm & s^dIzəm & sædıd్dzəm & sædəizəm & s^dIzəm & se I I I zəm \\
\hline saloon & Jalu:n & sılu:n & sælu:n & sælu:n & sılu:n & səlu:n \\
\hline said & $\int æ t$ & $s \wedge d$ & sæd & sæd & $\mathrm{s} \wedge \mathrm{d}$ & sed \\
\hline saturate & Jisureit & sıd్dzortt & sæt]u:reit & 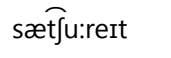 & sıdzorit & sætJəre It \\
\hline school & I $\int k u: l$ & Isku:I & Isku:I & sku:l & Isku:I & sku:I \\
\hline selection & fIlekJən & silıkJən & selekJən & selekJən & sılıkJən & silek $\int \mathrm{n}$ \\
\hline selfless & Selphles & SIIflis & selfles & selfles & SIIflis & selfl I s \\
\hline series & stri: $\widehat{d 3}$ & sIri:z & si:ri:z/dz & si:ri:z & sIri:z & S I əri:z \\
\hline serious & $\int \operatorname{Irr} \partial \int$ & SIrINS & si:rrəs & si:rıəs & SIrINS & siəriəs \\
\hline session & Jejən & $\operatorname{SI} \int \wedge n$ & seifən & seifən & $\mathrm{SI} / \wedge \mathrm{n}$ & $\operatorname{se} \int \mathrm{n}$ \\
\hline shortage & $\int \operatorname{artid} \widehat{d}$ & $\int \partial r t I \widehat{d z}$ & $\int a: r t I \widetilde{d} 3$ & Ja:rtid̋ & $\int \partial r t I \widehat{d z}$ & $\int 0: \operatorname{tid} 3$ \\
\hline shovel & $\int \nexists b^{h} \partial l$ & fa:fəl & Ja:vəl & Ja:vəl & Ja:fəl & $\int \Lambda \mathrm{vl}$ \\
\hline silent & farlent & sa:Int & sailent & sailent & sa:Innt & sa I lənt \\
\hline simple & $\int \mathrm{Imfəl}$ & simb^l & simpəl & simpəl & simbNl & s I mpl \\
\hline single & fIngəl & sinkəl & sInəl & sinəl & sinkəl & s I ggl \\
\hline finger & $\mathrm{p}^{h_{\text {IInger }}}$ & finger & finger & finger & finger & finger \\
\hline solace & fulei & soleis & səuleis & səuleIs & soleis & splis \\
\hline stopped & fta:pd & stupid & fta:pd & sta:pd & stupid & stopt \\
\hline tangible & tendżIbəl & tIndżżbəl & tendżIbəl & tend̄zIbəl & tInd̄żbəl & tænđక̧วbl \\
\hline territory & terituri & tIrrttri & teritərı & teritəri & trritiri & terətr I \\
\hline theft & ðер ${ }^{h t}$ & sIft & $\theta$ eft & Өeft & sIft & $\theta \mathrm{eft}$ \\
\hline thief & ði:ph & si:f & Өi:f & Өi:f & si:f & $\theta \mathrm{i}: \mathrm{f}$ \\
\hline thigh & ðаI & saI & $\theta a I$ & $\theta a I$ & saI & $\theta \mathrm{a}_{\mathrm{I}}$ \\
\hline
\end{tabular}




\begin{tabular}{|c|c|c|c|c|c|c|}
\hline throat & ðrəut & srəut & Orəut & Arəut & srəut & $\theta$ raot \\
\hline through & ðru: & sru: & Өru: & Өru: & sru: & $\theta$ ru: \\
\hline thumb & ðәmb & $\mathrm{s} \wedge \mathrm{mb}$ & ðәmb & ðәmb & $\mathrm{s} \wedge \mathrm{mb}$ & $\theta \Lambda \mathrm{m}$ \\
\hline tortoise & tartə] & tərtəs & ta:rtəs & ta:rtəs & tərtəs & to:təs \\
\hline towards & towərtd] & tuwsrdz & towərdz & towərdz & tuwsrdz & təwว:dz \\
\hline tradition & tratıfən & trədIIən & treidisən & treidIfən & trədIfən & $\operatorname{tr} \partial \mathrm{d} I \int \mathrm{n}$ \\
\hline transfer & tra:nffər & trənsfər & tra:nspər & tra:nsfər & trənsfər & trænsf3:(r) \\
\hline trigger & traigər & trigər & traigər & traigər & trigər & $\operatorname{tr} I g \partial(r)$ \\
\hline ultimate & ^lti:mæt & ^ltımet & ^ltımeit & $\Lambda$ ltımeit & Nltimet & slt I mat \\
\hline uncertain & ^nfərtən & ^nsərtin & ^nsərtən & ^nsərtən & ^nsərtın & $\Lambda \mathrm{ns} 3: \mathrm{tn}$ \\
\hline usual & ju:zvəl & jvfचəl & ju:jचəl & ju:jचəl & 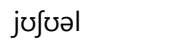 & ju:3l \\
\hline usurp & ju:đ̄zərp & juzərb & ju:sərp & ju:sərp & juzərb & ju:zz:p \\
\hline verdant & $b^{\text {h }}$ rtent & fərdInt & vərdent & vərdent & fərdint & vз:dnt \\
\hline victual & bıksuəl & fIkdَ̋ðəl & viktfuəl & viktfuəl & fıkd̋zoəl & vitl \\
\hline vigilant & bıฮ̋zIlent & fidzIIInt & vidz̧i:lent & vidżi:lent & frôdzIIInt & vi đ3̈ I lənt \\
\hline vision & vi:zən & vIfən & vi:zən & VIJən & VIJən & vi $3 n$ \\
\hline wastage & bestid $\widehat{d}$ & fistId $\widehat{d 3}$ & weIstid $\widehat{3}$ & weistid $\widehat{3}$ & fistid] & weistidz \\
\hline we & $b^{\text {h}} U I$ & $f_{I}$ & woi: & woi: & $\mathrm{fI}_{\mathrm{I}}$ & wi: \\
\hline wear & biər & fiər & wi:ər & wi:ər & fiər & weว(r) \\
\hline weather & beidər & fizər & weIðər & weIðər & fizər & weðə(r) \\
\hline wages & beidz & f/weig & weidz & weids & weids & weidz \\
\hline whistle & $b^{h_{1}} I \partial l$ & fisəl & WIsəl & Wisəl & fisəl & wisl \\
\hline Word & Bangladesh & Egypt & India & Pakistan & Saudi Arabia & Natives \\
\hline worth & $b^{\text {hərð }}$ & fərð & wər $\theta$ & wər$\theta$ & fərð & шз: $\theta$ \\
\hline wrath & rað & rað & ra: $\theta$ & ra: $\theta$ & rað & $\mathrm{ro} / æ \theta$ \\
\hline wrinkle & rinkəl & rinkəl & rinkəl & rinkəl & rinkəl & rigkl \\
\hline wry & raI & ri: & ri: & ri: & ri: & $\mathrm{raI}$ \\
\hline xerox & dzIrəovks & zIrəokis & dzIrəuks & zirəovs & zIrəokis & zi əroks \\
\hline yacht & ja:s & ja: & ja:t؟ & ja:t厅 & ja: $\int$ & $\mathrm{jpt}$ \\
\hline youth & ju:s & ju:s & ju: $\theta$ & ju: $\theta$ & ju:s & ju: $\theta$ \\
\hline zenith & $\widetilde{d}$ zInIt & zi:nis & zi:nə $\theta$ & zi:nə $\theta$ & zi:nis & $\operatorname{zen} I \theta$ \\
\hline
\end{tabular}

\section{Copyrights}

Copyright for this article is retained by the author(s), with first publication rights granted to the journal.

This is an open-access article distributed under the terms and conditions of the Creative Commons Attribution license (http://creativecommons.org/licenses/by/3.0/). 